\title{
Overexpression of RAB34 correlates with poor prognosis and tumor progression in hepatocellular carcinoma
}

\author{
JIANWU WU*, YIJIE LU* , ANCHENG QIN, ZHIMING QIAO and XINWEI JIANG \\ Department of General Surgery, Nanjing Medical University, Affiliated Suzhou Hospital, \\ Gusu, Suzhou, Jiangsu 215000, P.R. China
}

Received March 21, 2017; Accepted August 22, 2017

DOI: 10.3892/or.2017.5957

\begin{abstract}
RAB34, a protein belonging to the RAB family, is involved in protein transport, repositioning of lysosomes and activation of micropinocytosis. However, few studies have reported its function in human epithelial cancers. Immunohistochemistry (IHC) and western blotting were used to detect expression of RAB34 at the tissue and cell levels. Cell Counting Kit-8 (CCK-8), EDU assay and flow cytometry were used for analyzing cell proliferation. Transwell and scratch wound healing assays were used for assessing cell migration ability. Western blotting was used for detecting expression of E-cadherin and N-cadherin. In the present study, we found that both DNA copy and protein level of RAB34 were upregulating in human hepatocellular carcinoma (HCC) tissues when compared with that in adjacent tissues. Analysis of the correlation between RAB34 expression and clinicopathological features showed that patients with overexpression of RAB34 consistently had large tumor size, vessel invasion and poor tumor grade. Furthermore, overall survival analysis showed that patients with upregulated expression of RAB34 were associated with poor prognosis. Moreover, cell function experiments showed that suppression of RAB34 led to a lower proliferation rate and migration ability. In addition, this phenomenon may be attributed to cell cycle phase G1 arrest and mesenchymal-epithelial transition under condition of RAB34 suppression. The present study demonstrated that RAB34 plays an important role in the initiation and progression of HCC. Our results suggest a new therapeutic target for the clinical treatment of HCC.
\end{abstract}

Correspondence to: Dr Xinwei Jiang, Department of General Surgery, Suzhou Municipal Hospital, 26 Daoqian Street, Gusu, Suzhou, Jiangsu 215000, P.R. China

E-mail: jiangxinweimm@sina.com

${ }^{*}$ Contributed equally

Key words: RAB34, hepatocellular carcinoma, mesenchymalepithelial transition, proliferation, migration, prognosis

\section{Introduction}

Hepatocellular carcinoma (HCC), a common malignancy worldwide, is associated with persistently increasing incidence and mortality rates (1). Patients with early stage HCC are indicated for multiple treatments including surgical resection, liver transplantation or local ablation, and are associated with a better prognosis. However, the sole strategy for patients with intermediate and advanced HCC is chemotherapy to inhibit tumor progression and these patients are associated with a poor prognosis (2). Thus, developing methods to facilitate the diagnosis of early stage HCC can improve the prognosis of these patients. However, the detection of existing tumor biomarkers is unable to meet the demand (3). Thus, we need to identify new tumor biomarkers for facilitating the early detection of HCC.

$R A B 34$ is a gene that encodes a protein belonging to the RAB family of proteins, which are small GTPases involved in protein transport. Wang and colleagues found that RAB34 is involved in the repositioning of lysosomes and the activation of micropinocytosis by interacting with Rab-interacting lysosomal protein (RILP) (4-6). Further study by Starling et al showed that the formation of the RAB34-PILP complex is regulated by folliculin under the condition of nutrients (7). In addition, Speight and Silverman found that RAB34 is also regulated by Hmunc13, which is a cytosolic diacylglycerol (DAG)-binding protein (8). Alloatti et al showed that RAB34 plays an important role in the topological re-organization of lysosomes during a transient phase after TLR4 engagement in the maturation of dendritic cells (9). A previous study showed that Rab34/Munc13-2 plays an important role in alternative Rab7-independent phagosome maturation. This result uncovered a deeper mechanism of RAB34 in regulating phagolysosome fusion (10). In addition to these findings, Wang et al demonstrated that overexpression of RAB34 is related to the progression of glioma grade and is associated with the poor prognosis of high-grade glioma patients (11). Based on these findings, we aimed to ascertain whether RAB34 plays a vital role in the prognosis of HCC patients.

In the present study, we first found that RAB34 was overexpressed in HCC tissues, and was correlated with tumor size and tumor grade. Further overall survival analysis showed that patients with high expression of RAB34 consistently had poor prognosis. Then, we used siRNA to study the function 
of RAB34 in HCC cell lines. We found that suppression of RAB34 led to a decrease in the cell proliferation rate and migration ability. Further study showed that RAB34 regulated the progression of the G1 phase in the cell cycle and epithelial to mesenchymal transition. In conclusion, we found that RAB34 plays a vital role in the progression of $\mathrm{HCC}$ and may be a new biomarker for assessing the prognosis of HCC patients.

\section{Materials and methods}

Patients, tissue samples and follow-up. All cancer and adjacent tissue samples were obtained from 79 HCC patients who underwent surgery at the Nanjing Medical University Affiliated Suzhou Hospital from September 1, 2012 to September 1, 2013. All patients were diagnosed with HCC by imaging and serological examination and accepted surgery without radiation and chemotherapy. Fresh cancer and adjacent matched normal tissues were collected and used for constructing paraffin blocks. Two pathologists examined the cancer and the matched normal tissues. The Medical Ethics Committee of The Affiliated Suzhou Hospital of Nanjing Medical University approved the protocol, and written informed consent was obtained from all participants. Clinical data of all the HCC patients including age, sex, size of the tumor, number of tumors, vessel invasion and tumor grade were collected. Two students were responsible for the follow-up of these patients every 3 months. All of these patients were followed up until the follow-up termination date (July 31,2016 ) or death. Overall survival was calculated in months from the diagnosis until the date of death, last known to be alive, or the study closing date.

Ethical approval. The Second Affiliated Hospital, Xi'an Jiaotong University Ethics Committee approved the present study. Informed consent was obtained from all individual participants included in the study. All procedures performed in studies involving human participants were in accordance with the ethical standards of the institutional and/or national research committee and with the 1964 Helsinki Declaration and its later amendments or comparable ethical standards.

Cell culture. HCC cell lines LM3, Huh7, SK and 7701 were purchased from the Chinese Academy of Science Cell Bank (Shanghai, China). All cells were maintained in minimum essential medium (MEM), and supplemented with $10 \%$ fetal bovine serum (FBS) at $37^{\circ} \mathrm{C}$ in a humidified atmosphere of $5 \% \mathrm{CO}_{2}$.

Transfection of LM3 and Huh7 cell lines with small interfering $R N A$. Human-specific RAB34 small interfering RNA (siRNA) was designed, constructed and purified by Jima Biotech Co. (Shanghai, China). siRNA targeting Rab34 had the following annealed duplex: 5'-AAUCGUUCCAUCUCGAAGUCCA CUC-3' and 5-'GAGUGGACUUCGAGAUGGAACGAUU-3'. The transfection was conducted using Lipofectamine 2000 (Invitrogen, Carlsbad, CA, USA) according to the manufacturer's instructions.

Immunohistochemistry (IHC). The 79 paired paraffinembedded cancer and adjacent tissues of HCC patients were sectioned $(3 \mu \mathrm{m})$, deparaffinized in xylene, and rehydrated in a series of graded alcohol dilutions. Then, we used heat epitope retrieval for 20 min under a condition of citrate salt solution. After incubation in 5\% BSA for $30 \mathrm{~min}$, all tissues were incubated with a rabbit antibody to human RAB34 anti-RAB34 antibody (ab110821; dilution, 1/1,000; Abcam Biotechnology, Cambridge, UK) overnight at $4^{\circ} \mathrm{C}$. Slides were then incubated with HRP at room temperature for $30 \mathrm{~min}$ and were visualized using DAB as chromogen for 5-10 min. The method for IHC scoring was as follows: 0 , negative; $1,<30 \%$ positive tumor cells; $2,30-50 \%$ positive tumor cells; $3,50-70 \%$ positive tumor cells; and $4,>70 \%$ positive tumor cells. The intensity of the dye color was graded as 0 (no color), 1 (light yellow), 2 (light brown), or 3 (brown). The staining index was calculated as follows: Staining index $=$ staining intensity + tumor cell staining grade. High RAB34 expression was defined as a staining index score $\geq 4$, while low expression was defined as a staining index $<4$.

Western blot analysis. Total proteins were extracted from the harvested cells using a protein extraction kit (Beyotime, Jiangsu, China). All the proteins were degenerated by boiling, separated by SDS-PAGE and transferred onto polyvinylidene difluoride (PVDF) membranes. Then, the membranes were blocked by $5 \%$ milk. Next, the membranes were incubated in the primary antibodies overnight at $4^{\circ} \mathrm{C}$. Then, after washing 3 times with Tris-buffered saline and Tween-20 (TBST), the membranes were incubated with the secondary antibody (goat anti-rabbit) for $2 \mathrm{~h}$ in a $37^{\circ} \mathrm{C}$ incubator. Finally, all the membranes were visualized using a chemiluminescence kit (Beyotime) on a Bio-Rad imaging system. Primary antibodies used in the present study were as follows: RAB34 (ab110821; dilution, 1/1,000; Abcam Biotechnology), E-cadherin and $\mathrm{N}$-cadherin [1:1,000 dilution, (EMT) Antibody Sampler kit \#9782; Cell Signaling Technology, Beverly, MA, USA], CDK-2 (ab32147; dilution, 1/1,000), CDK4 (ab108357; dilution, 1/1,000) and cyclin B1 (ab32053; dilution, 1/1,000) and the internal control was $\beta$-actin (dilution, 1:1,000) (all from Abcam Biotechnology).

Cell viability and EDU assays. To detect the relative cell viability, LM3 and Huh7 cell lines were seeded into 96-well microplates at a density of 5,000 cells/well. Then, Cell Counting Kit-8 (CCK-8) assay (Dojindo, Kumamoto, Japan) was performed according to the manufacturer's instructions at 24,48 and $72 \mathrm{~h}$. Briefly, $10 \mu \mathrm{l}$ of CCK-8 working solution/ $100 \mu \mathrm{l}$ of medium was added into the microplates and the cells were incubated for $2 \mathrm{~h}$. The optical density OD450 value was determined using an MRX II microplate reader (DYNEX Technologies, Chantilly, VA, USA). In addition, the detection of EDU was conducted according to a previous study (12).

Cell cycle analysis. LM3 and Huh7 cells transfected with the RAB34 siRNA or the negative control (NC) were trypsinized and washed 3 times with pre-chilled phosphate-buffered saline (PBS), and resuspended in $100 \mu \mathrm{l}$ PBS at $1 \times 10^{6}$ cells $/ \mathrm{ml}$ after $48 \mathrm{~h}$ of transfection. For cell cycle analysis, samples of cells were fixed in 75\% ethanol overnight, and DNA was stained for cell cycle analysis. RNA was removed by mixing $0.5 \mathrm{ml}$ DNA Prep Stain (Coulter DNA Prep Reagents kit; Beckman Coulter, Brea, CA, USA) in dark at room temperature for 
Table I. Relationship between RAB34 expression and clinicopathologic features of the HCC patients.

\begin{tabular}{|c|c|c|c|c|}
\hline \multirow[b]{2}{*}{ Variables } & \multirow{2}{*}{$\frac{\text { All pts. }}{(n=79)}$} & \multicolumn{2}{|c|}{$\begin{array}{c}\text { RAB34 } \\
\text { expression }\end{array}$} & \multirow[b]{2}{*}{ P-value } \\
\hline & & $\operatorname{High}^{\mathrm{a}}$ & Low $^{\text {b }}$ & \\
\hline Age (years) & & & & 0.783 \\
\hline$\leq 55$ & 37 & 20 & 17 & \\
\hline$>55$ & 42 & 24 & 18 & \\
\hline Gender & & & & 0.822 \\
\hline Male & 35 & 19 & 16 & \\
\hline Female & 44 & 25 & 19 & \\
\hline Size of tumor $(\mathrm{cm})$ & & & & 0.024 \\
\hline$\leq 5$ & 34 & 14 & 20 & \\
\hline$>5$ & 45 & 30 & 15 & \\
\hline Number of tumors & & & & 0.143 \\
\hline Single & 47 & 23 & 24 & \\
\hline Multiple & 32 & 21 & 11 & \\
\hline Vessel invasion & & & & 0.115 \\
\hline Negative & 49 & 25 & 24 & \\
\hline Positive & 30 & 19 & 11 & \\
\hline Grade & & & & 0.026 \\
\hline Well + moderate & 32 & 13 & 19 & \\
\hline Poor & 47 & 31 & 16 & \\
\hline
\end{tabular}

${ }^{a}$ High, IHC score $\geq 4$; blow, IHC score $<4$. pts., patients. For analysis of correlation between RAB34 expression and clinical features, Chi-square tests were used. Results were considered statistically significant at ${ }^{\mathrm{c}} \mathrm{P}<0.05$.

30 min. DNA content of stained cells was measured with BD LSR II (BD Biosciences, Bedford, MA, USA). Each histogram was constructed with data from at least 10,000 events. The percentage of the cell population in each phase from the experimental data was calculated using ModFit LT software (Verity Software House, Inc., Topsham, ME, USA).

Cell migration assay. Migration assays were performed using Transwell plates (Corning, Corning, NY, USA). After $48 \mathrm{~h}$ of transfection, LM3 and Huh7 cells transfected with RAB34 siRNA or the negative control were trypsinized and collected. Then, $1 \times 10^{5}$ cells were cultured in serum-free MEM on an insert coated without Matrigel (migration assay; BD Biosciences) for $24 \mathrm{~h}$. In the lower compartment, medium was replaced with MEM complete medium. After fixation and staining, cells on the bottom surface that invaded across the membranes were counted and photographed. All experiments were performed in triplicate.

Scratch wound healing assay. LM3 and Huh7 cells transfected with RAB34 siRNA or the negative control were inoculated onto 6-well plates and cultured at $37^{\circ} \mathrm{C}$ in a $5 \% \mathrm{CO}_{2}$ cell incubator. After the cells reached $70-80 \%$ confluence, cross lines were made using a $200-\mu 1$ sterile pipette tip. The cells were washed 3 times with sterile PBS to remove the scratched cells. The cells were continuously cultured in serum-free culture medium. After 0 and 48 h, the cells were photographed. Cell migration distance $=$ distance at $0 \mathrm{~h}$ - distance at $48 \mathrm{~h}$.

Statistical analysis. Data are presented as means \pm standard deviation (SD). For comparisons, the Student's t-test, paired-samples t-test, and Fisher's exact test were performed as appropriate. Cumulative recurrence and survival probabilities were evaluated using the Kaplan-Meier method, and differences were assessed using the log-rank test. All analyses were performed using SPSS 18.0 software (SPSS, Inc., Chicago, IL, USA). Differences were considered significant at $\mathrm{P}<0.05$.

\section{Results}

RAB34 is upregulated in human HCC tissues and cell lines. To investigate whether RAB34 plays an important role in the initiation and progression of HCC, we detected the expression level of RAB34 by IHC in 79 paired cancer and adjacent tissues of HCC patients. The results showed that RAB34 was overexpressed in almost all HCC tissues. By comparing with the neighbor-cancer tissues, RAB34 was upregulated in 52 HCC tissues, while downregulated in only 13 (Fig. 1A). To verify our findings, we searched Oncomine database using the items ('RAB34' and 'liver cancer vs. normal') and found the data of RAB34 copy no. in 212 TCGA liver tissues, which showed that the RAB34 DNA copy no. was overexpressed in HCC (Fig. 1B). Furthermore, we compared the RAB34 expression level among 3 HCC cell lines (Huh7, SK and LM3) and a normal immortalized liver cell line (7701), and found that RAB34 was overexpressed in the HCC cell lines, and the LM3 cell line showed the highest expression (Fig. 1C). Such results showed that RAB34 was upregulated in HCC and may be associated with the initiation and progression of HCC.

Overexpression of RAB34 leads to the poor prognosis of HCC patients. To investigate whether RAB34 expression is associated with the prognosis of HCC patients, we divided the HCC patients into two groups according to the expression of RAB34 in cancer tissues (44 patients were high expression group while 35 patients were low expression group). The results of Kaplan-Meier analysis showed that the patients with high expression of RAB34 were associated with poor prognosis when compared with those who had low expression $(\mathrm{P}=0.026)$ (Fig. 2). In addition, we analyzed the relationship between RAB34 expression and clinicopathological features of HCC patients by single-factor Chi-square test. We found a correlation between expression of RAB34 and tumor size and tumor grade (Table I). Patients with high expression of RAB34 consistently had large tumor size and poor tumor grade.

Suppression of RAB34 inhibits the proliferation of HCC-LM3 and Huh7 cell lines. As expression of RAB34 in Huh7 and HCC-LM3 cells was relative higher than that noted in the SK cell line, and SK was derived from ascites, Huh7 and HCC-LM3 cells were deemed suitable for our further study. We used RAB34 siRNA to suppress the expression of RAB34 in the HCC-LM3 and Huh7 cell lines, and the efficiency of 

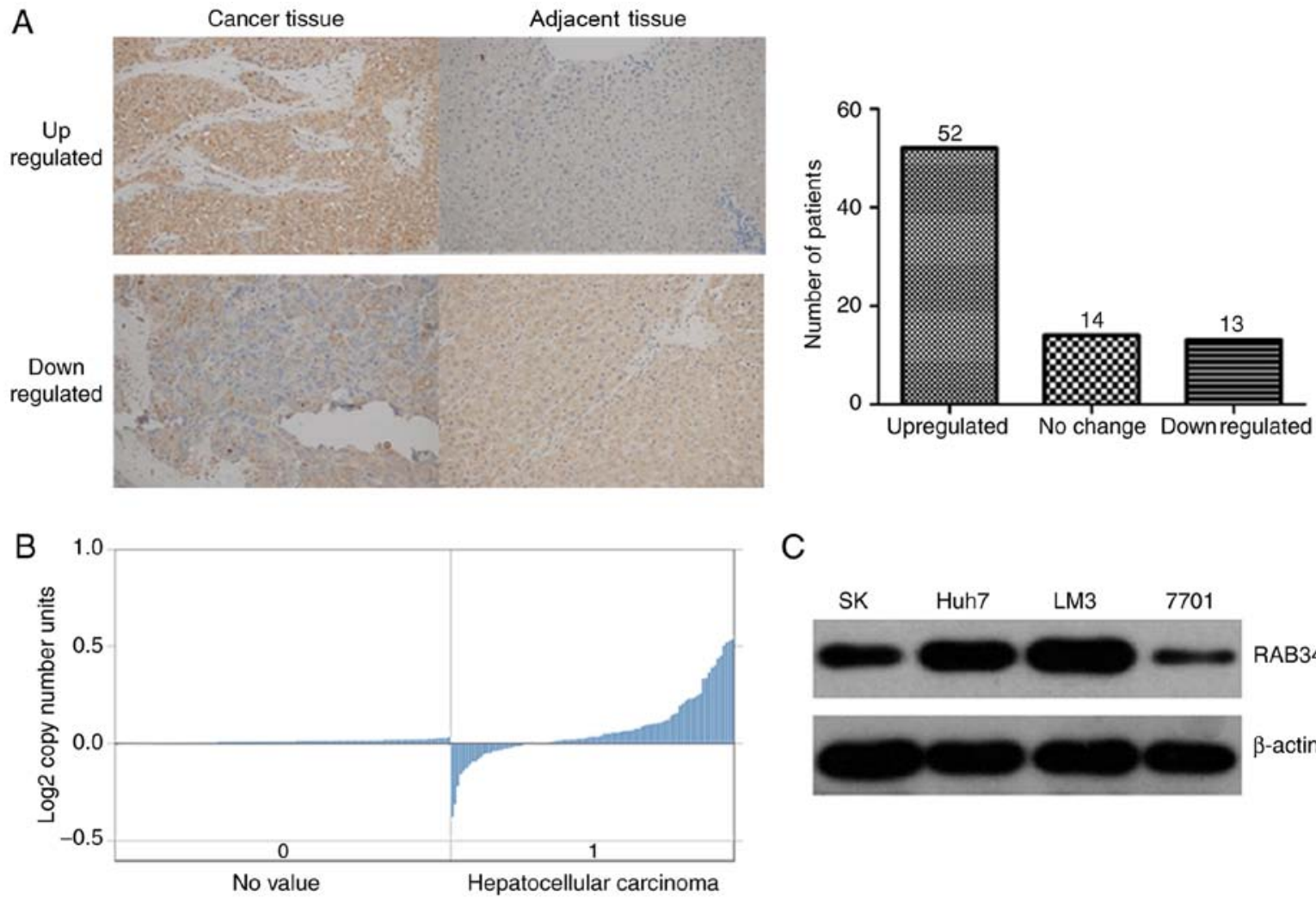

C

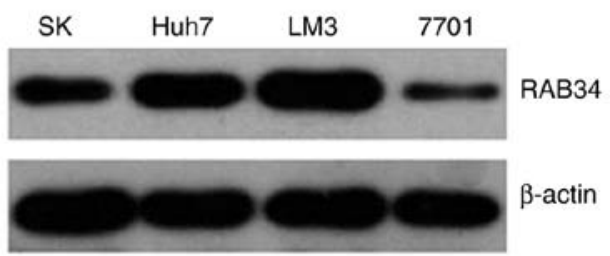

Figure 1. (A) Expression of RAB34 in 79 paired HCC cancer and adjacent tissues, and the results are quantified using a histogram. (B) The comparison between liver and HCC tissues in regards to the number of RAB34 DNA copies. The results are from the Oncomine database. (C) Expression of RAB34 in SK, Huh7, LM3 and 7701 cell lines. $\beta$-actin was used as the internal control.

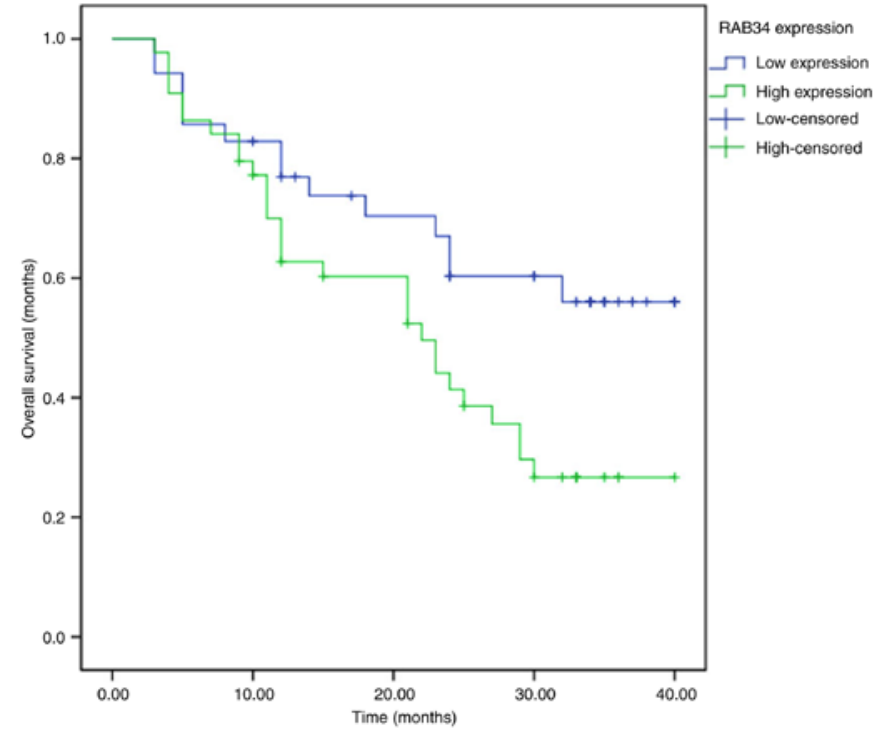

Figure 2. Results of the overall survival analysis of HCC patients according to tumors with high and low expression of RAB34.

knockdown was verified by western blotting (Fig. 3A). Then, we used CCK-8 assay to compared cell viability under condition of RAB34 siRNA and negative control, and found thtat the cell lines with RAB34 siRNA had lower cell viability than that noted in the negative controls (Fig. 3B). However, such a phenomenon may be induced by cell apoptosis. Thus, we detected the cell proliferation rate by EDU assay following transfection with the siRNA and negative control. The results showed that suppression of RAB34 markedly decreased the cell proliferation rate (Fig. 3C). Thus, we concluded that RAB34 regulated the proliferation of HCC-LM3 and Huh7 cell lines, and these results corroborated the results of the clinical sample analysis.

RAB34 affects the proliferation rate of HCC-LM3 and Huh7 cell lines by regulating progression of G1 cell cycle phase. To study the mechanism of RAB34 in regulating the proliferation rate of HCC cell lines, we used flow cytometry to detect cell cycle change in the cells transfected with the RAB34 siRNA and negative control. The results were analyzed by Modfit software and different cell cycle phases (G1, S and G2) were distinguished (Fig. 4A). Then, we used Graphpad software to compare the results of the two groups and found that the cell number in the G1 phase in the siRNA group was higher than that in the negative group, while the cell numbers in the $S$ and G2 phases were less than those in the negative group (Fig. 4B). Thus, suppression of RAB34 was found to lead to cell cycle G1 phase arrest. To further verify our findings, we used western blotting to detect expression of cell cycle-related proteins and found that CDK2, CDK4 and cyclin B1 were all decreased in the RAB34 siRNA group (Fig. 4C). Thus, we concluded that RAB34 positively regulates progression of cell cycle G1 phase.

Suppression of RAB34 inhibits the migration of HCC cell lines by mesenchymal-epithelial transition. Since suppression of 
A

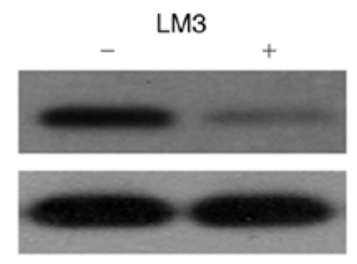

LM3

$\mathrm{B}$

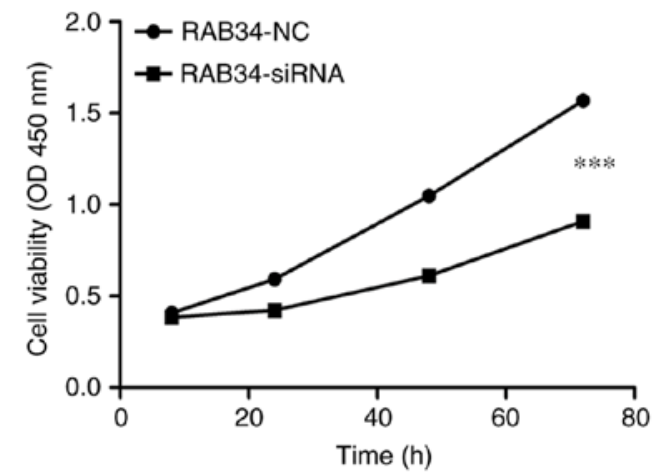

C
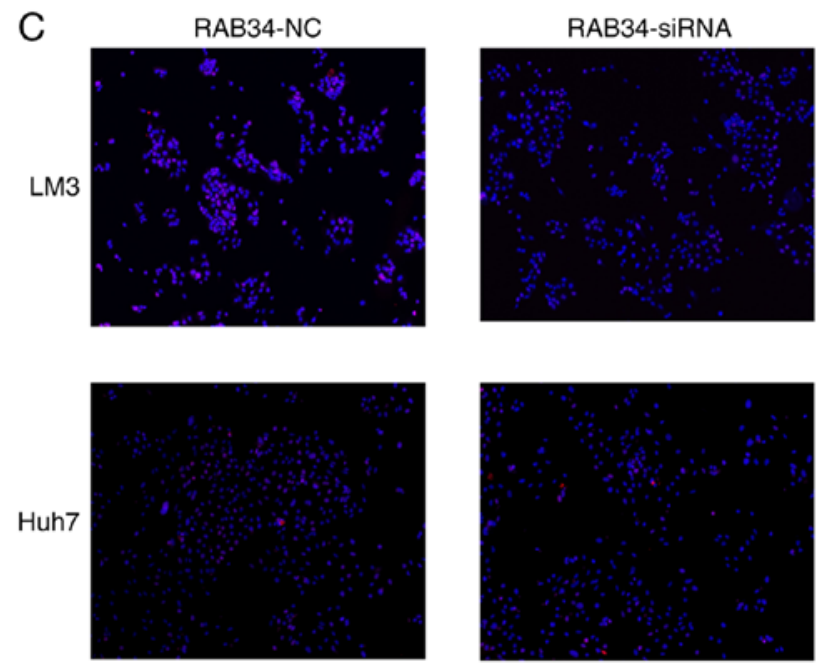
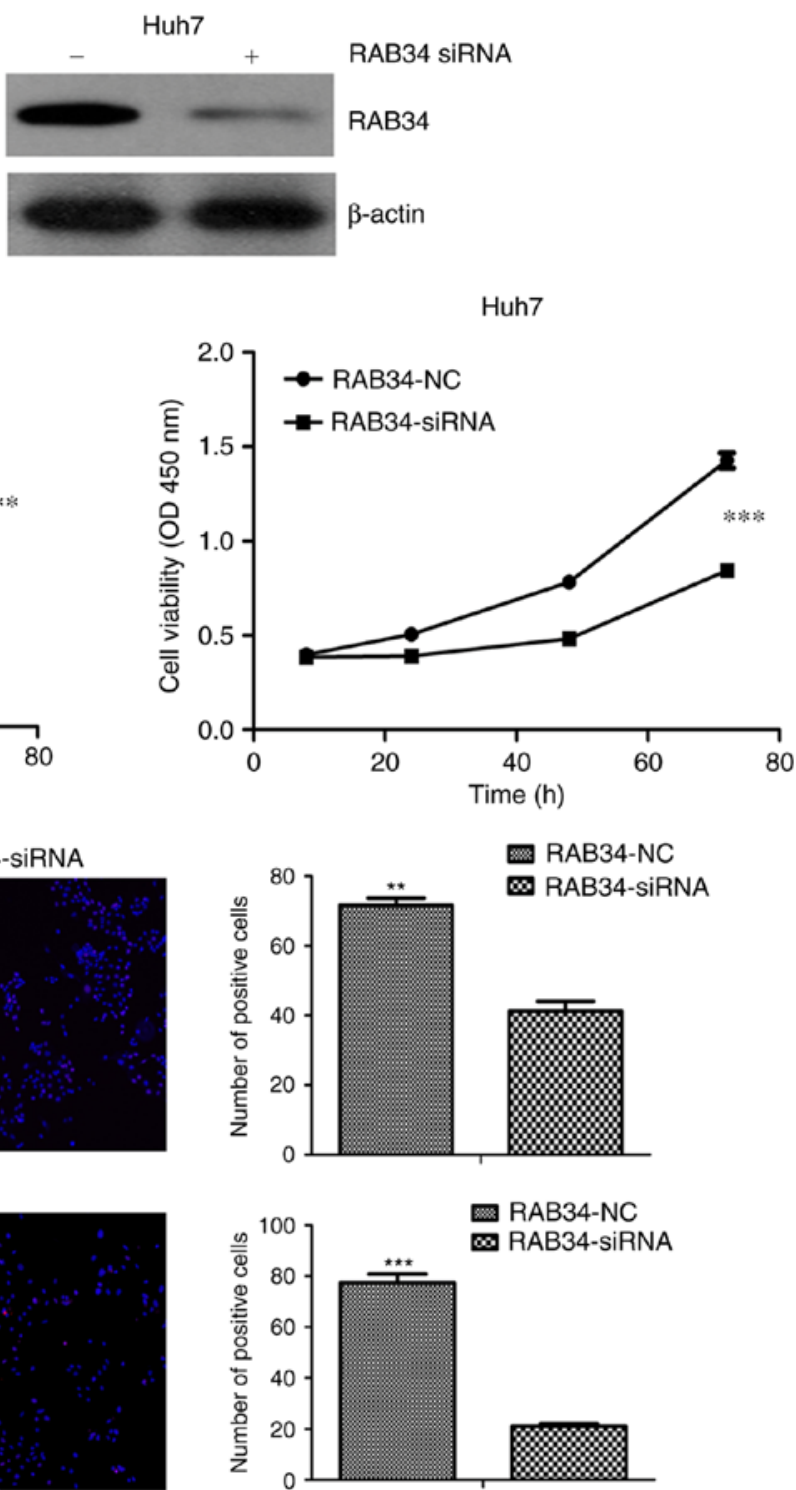

Figure 3. (A) Efficiency of RAB34 siRNA in LM3 and Huh7 cell lines. (B) Results of the CCK-8 assay in LM3 and Huh7 cell lines transfected with the RAB34 siRNA or negative control (NC); each group had 7 repeated data. (C) Results of the EDU assay in LM3 and Huh7 cell lines transfected with RAB34 siRNA or $\mathrm{NC}$, and the results were quantified; ${ }^{* * * *} \mathrm{P}<0.0001$.

RAB34 regulated the proliferation rate of HCC cell lines by inhibiting cell cycle progression, we aimed to ascertain whether it plays an important role in cell migration. We first used the Transwell assay to detect cell migration change in the cells following transfection of RAB34 siRNA or negative control. We found that the number of migrated cells in the siRNA groups was less than that noted in the negative controls (Fig. 5A). To eliminate the interference of cell proliferation, we used a scratch wound healing assay to detect cell migration ability under a serum-free environment and found the same result as above (Fig. 5B). Thus, suppression of RAB34 inhibited the ability of cell migration. To study the mechanism of such a phenomenon, we used western blotting to detect expression of E-cadherin and N-cadherin in the RAB34 siRNA and negative groups. The results showed that suppression of RAB34 led to higher expression of E-cadherin and lower expression of $\mathrm{N}$-cadherin when compared to the negative controls (Fig. 5C). Thus, we concluded that suppression of RAB34 inhibited cell migration by mesenchymal-epithelial transition.

\section{Discussion}

The Ras superfamily is a protein superfamily of small GTPases, which are divided into 5 main families (Ras, Rho, Ran, Rab and Arf) based on their structure, sequence and function $(13,14)$. Numerous studies have shown that vesicle trafficking and exocytosis are important in tumorigenesis, which suggests that abnormal expression of RAB family proteins plays a vital role in the initiation and progression of tumors (15-17). In addition, numerous studies have shown that the dysregulation of the RAB family of proteins could be tumorigenic or tumor suppressive depending on different tumor microenvironments. Tong and colleagues found that RAB25 had low expression in esophageal squamous cell carcinoma and was an important tumor suppressor with both anti-invasive and -angiogenic abilities (18). Dong et al analyzed the overall survival of $148 \mathrm{HCC}$ patients and found that expression of Rab27A/B was correlated with clinicopathological characteristics and poor prognosis in HCC (19). In addition, a recent study found that 

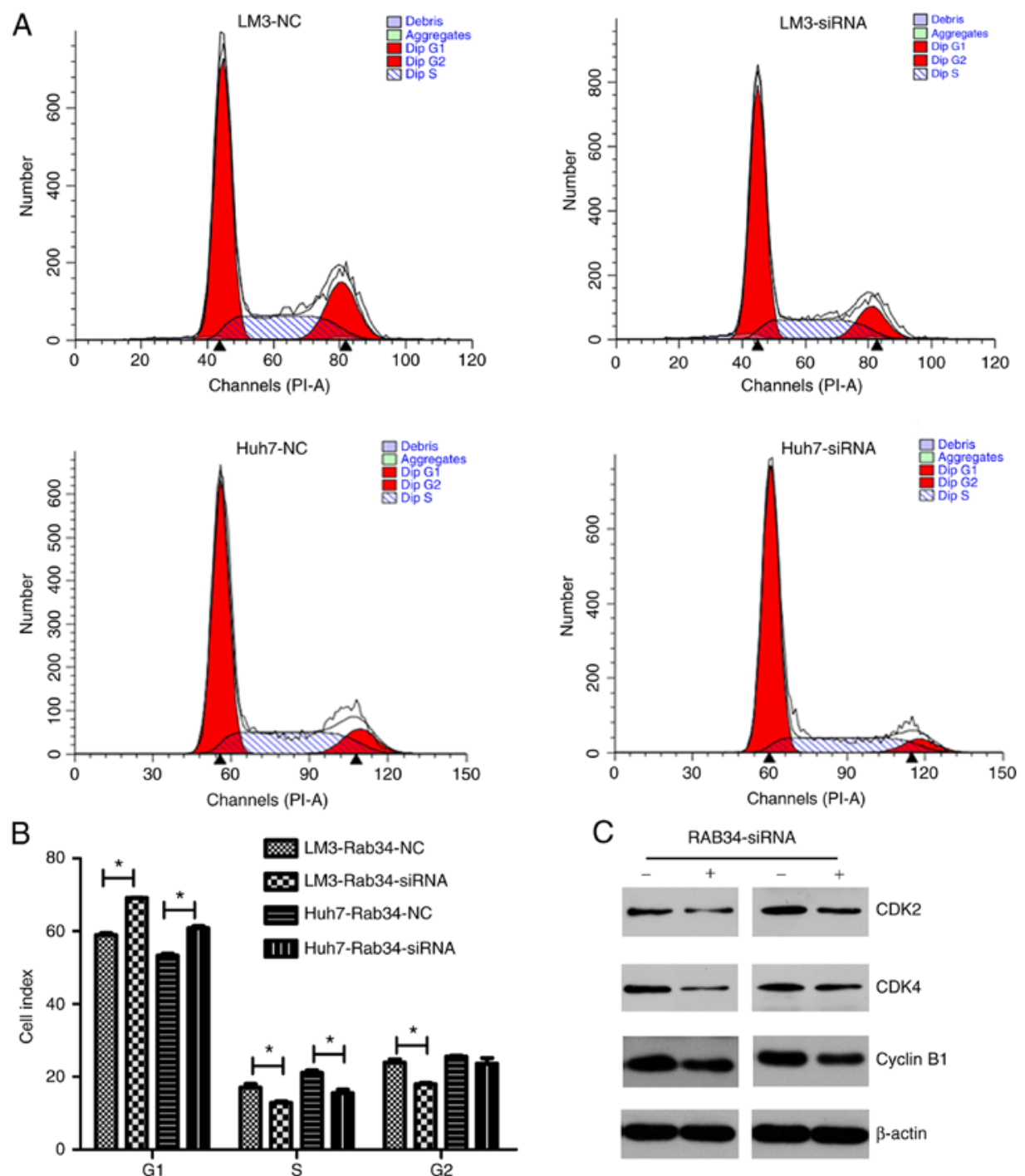

Figure 4. (A) Results of cell cycle analysis in LM3 and Huh7 cell lines transfected with RAB34 siRNA and negative control (NC), respectively. (B) The quantified results of cell cycle analysis; each group consisted of 3 experiments; ${ }^{*} \mathrm{P}<0.01$. (C) Expression change in CDK2, CDK4 and cyclin B1 in cells transfected with the RAB34 siRNA and NC. $\beta$-actin was used as the internal control.

RAB34 is associated with the progression of glioma grade and leads to poor prognosis in high-grade glioma patients (11). While the function of RAB34 in HCC was unclear, we aimed to ascertain whether RAB34 plays an important role in the progression of HCC.

Firstly, we found that RAB34 was overexpressed in HCC cancer tissues by IHC detection and Oncomine database analysis. Then, we found that expression of RAB34 was correlated with tumor size and tumor grade and patients with high expression of RAB34 consistently had a poor prognosis. Further study showed that suppression of RAB34 regulated the proliferation rate of HCC cell lines by inhibiting progression of the G1 phase, and inhibited the migration ability by promoting progression of an epithelial cell phenotype.

In 2011, Hanahan and Weinberg wrote a study called 'Hallmarks of Cancer: The Next Generation'. They showed 6 hallmark capabilities of cancer and the ability of immortal proliferation and migration were included (20). Thus, RAB34 may be a new therapeutic target for the clinical treatment of HCC. As the cell cycle phase is important for cell proliferation, each phase has its own checkpoint, and only passing through the detection of this checkpoint can the cell proliferate for the next generation $(21,22)$. Thus, we detected expression of various G1 proteins under a condition of RAB34 suppression, and found that CDK2, CDK4 and cyclin B1 were decreased. In contrast, epithelial-mesenchymal transition (EMT) which is a reversible dynamic process during which epithelial cells gradually adopt structural and functional characteristics of mesenchymal cells, has gained much attention regarding metastatic dissemination (23-25). We used western blotting to observe the expression change of E-cadherin and $\mathrm{N}$-cadherin under condition of RAB34 siRNA. Notably, we found that $\mathrm{E}$-cadherin was upregulated and $\mathrm{N}$-cadherin was downregulated when RAB34 was suppression. In conclusion, we demonstrated that RAB34 may become a new biomarker and therapeutic target for HCC.

However, in consideration of the limitation of experimental technology, we did not research the more detailed mechanism involved in the function of RAB34 in HCC. Thus, further clinical research is warranted to verify the clinical value 
A
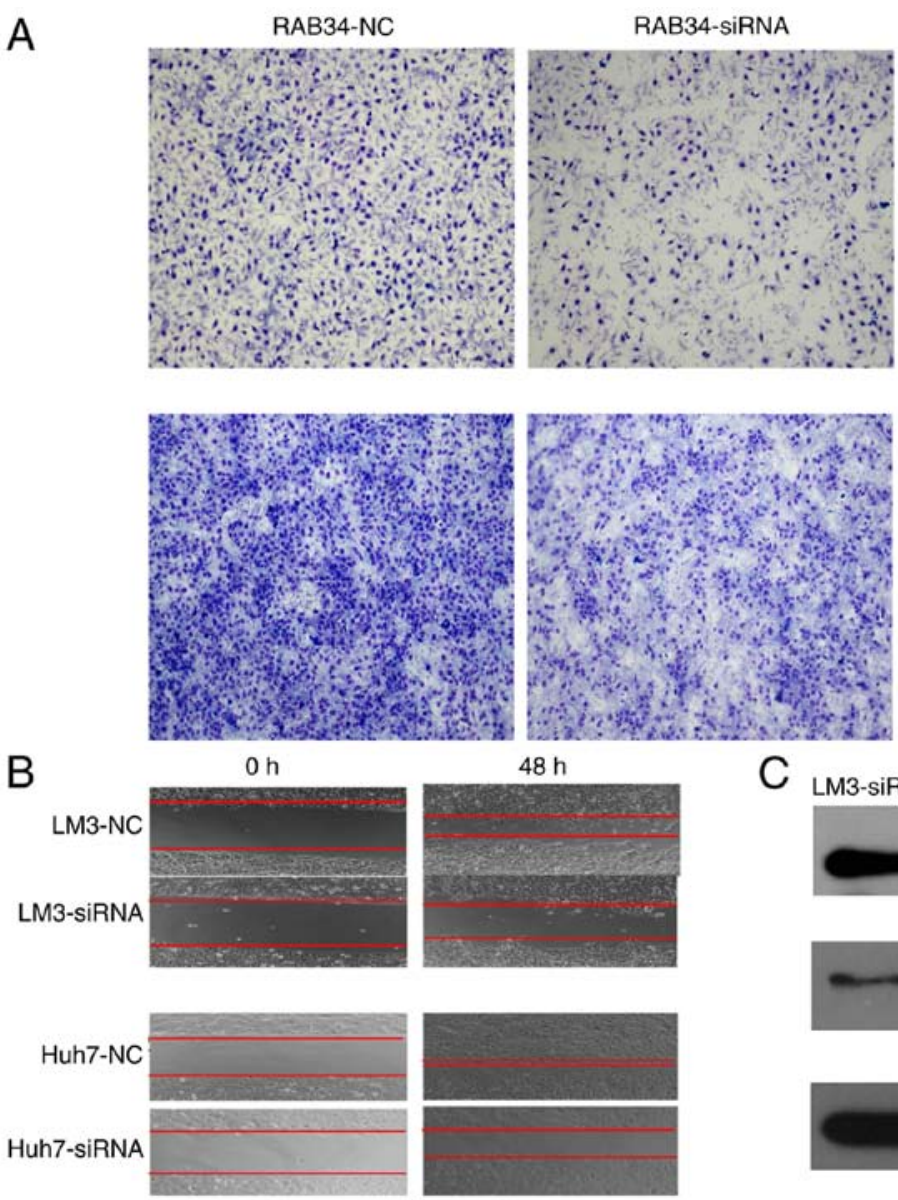

C

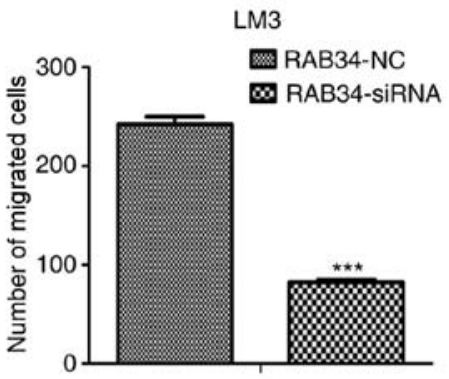

LM3-siRNA
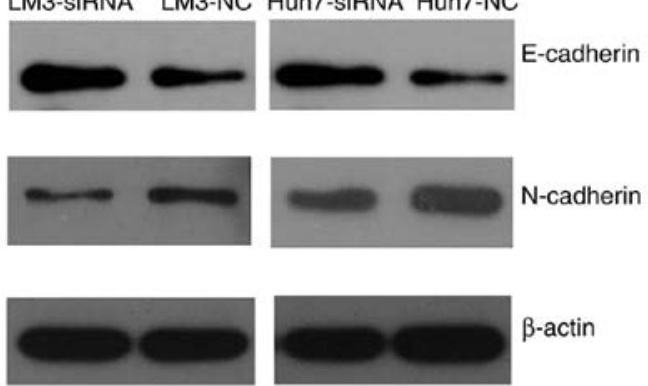

Figure 5. (A) Results of the Transwell assay in cells transfected with RAB34 siRNA and negative control (NC), and this result was quantified using histograms; ${ }^{* * * *} \mathrm{P}<0.0001$. (B) Results of the wound healing assay at 0 and $48 \mathrm{~h}$ following cell transfection of RAB34 siRNA and NC. (C) Results of western blotting show expression change in E-cadherin and N-cadherin under the condition of RAB34 siRNA and NC. $\beta$-actin was used as the internal control.

of RAB34. In a word, the present study demonstrated that RAB34 is overexpressed in HCC and plays an important role in the progression of $\mathrm{HCC}$; patients with high expression of RAB34 have a poor prognosis.

\section{References}

1. Finn RS: Current and future treatment strategies for patients with advanced hepatocellular carcinoma: Role of mTOR inhibition. Liver Cancer 1: 247-256, 2012.

2. Forner A, Gilabert M, Bruix J and Raoul JL: Treatment of intermediate-stage hepatocellular carcinoma. Nat Rev Clin Oncol 11: 525-535, 2014.

3. Galmiche A, Chauffert B and Barbare JC: New biological perspectives for the improvement of the efficacy of sorafenib in hepatocellular carcinoma. Cancer Lett 346: 159-162, 2014.

4. Wang $\mathrm{T}$ and Hong $\mathrm{W}$ : Interorganellar regulation of lysosome positioning by the Golgi apparatus through Rab34 interaction with Rab-interacting lysosomal protein. Mol Biol Cell 13: 4317-4332, 2002.

5. Wang T, Wong KK and Hong W: A unique region of RILP distinguishes it from its related proteins in its regulation of lysosomal morphology and interaction with Rab7 and Rab34. Mol Biol Cell 15: 815-826, 2004.

6. Wang T and Hong W: Assay and functional properties of Rab34 interaction with RILP in lysosome morphogenesis. In: Methods in Enzymology. Academic Press, New York, NY, pp675-687, 2005.

7. Starling GP, Yip YY, Sanger A, Morton PE, Eden ER and Dodding MP: Folliculin directs the formation of a Rab34-RILP complex to control the nutrient-dependent dynamic distribution of lysosomes. EMBO Rep 17: 823-841, 2016.
8. Speight $P$ and Silverman M: Diacylglycerol-activated Hmunc13 serves as an effector of the GTPase Rab34. Traffic 6: 858-865, 2005.

9. Alloatti A, Kotsias F, Pauwels AM, Carpier JM, Jouve M, Timmerman E, Pace L, Vargas P, Maurin M, Gehrmann U, et al: Toll-like receptor 4 engagement on dendritic cells restrains phago-lysosome fusion and promotes cross-presentation of antigens. Immunity 43: 1087-1100, 2015.

10. Kasmapour B, Gronow A, Bleck CK, Hong W and Gutierrez MG: Size-dependent mechanism of cargo sorting during lysosomephagosome fusion is controlled by Rab34. Proc Natl Acad Sci USA 109: 20485-20490, 2012.

11. Wang HJ, Gao Y, Chen L, Li YL and Jiang CL: RAB34 was a progression- and prognosis-associated biomarker in gliomas. Tumour Biol 36: 1573-1578, 2015.

12. Salic A and Mitchison TJ: A chemical method for fast and sensitive detection of DNA synthesis in vivo. Proc Natl Acad Sci USA 105: 2415-2420, 2008.

13. Goitre L: The Ras superfamily of small GTPases: The unlocked secrets. In: Ras Signaling: Methods and Protocols. Trabalzini L and Retta SF (eds). Humana Press, Totowa, NJ, pp1-18, 2014.

14. Wennerberg K, Rossman KL and Der CJ: The Ras superfamily at a glance. J Cell Sci 118: 843-846, 2005.

15. Chan AM and Weber T: A putative link between exocytosis and tumor development. Cancer Cell 2: 427-428, 2002.

16. Wright PK: Targeting vesicle trafficking: An important approach to cancer chemotherapy. Recent Patents Anticancer Drug Discov 3: 137-147, 2008.

17. Chia WJ and Tang BL: Emerging roles for Rab family GTPases in human cancer. Biochim Biophys Acta 1795: 110-116, 2009.

18. Tong M, Chan KW, Bao JY, Wong KY, Chen JN, Kwan PS, Tang KH, Fu L, Qin YR, Lok S, et al: Rab25 is a tumor suppressor gene with antiangiogenic and anti-invasive activities in esophageal squamous cell carcinoma. Cancer Res 72: 6024-6035, 2012. 
19. Dong WW, Mou Q, Chen J, Cui JT, Li WM and Xiao WH: Differential expression of Rab27A/B correlates with clinical outcome in hepatocellular carcinoma. World J Gastroenterol 18 : 1806-1813, 2012.

20. Hanahan D and Weinberg RA: Hallmarks of cancer: The next generation. Cell 144: 646-674, 2011.

21. Murray AW: Recycling the cell cycle: Cyclins revisited. Cell 116 221-234, 2004.

22. Sears RC and Nevins JR: Signaling networks that link cell proliferation and cell fate. J Biol Chem 277: 11617-11620, 2002.
23. Nieto MA and Cano A: The epithelial-mesenchymal transition under control: Global programs to regulate epithelial plasticity. Semin Cancer Biol 22: 361-368, 2012.

24. Guarino M,Rubino B and Ballabio G: The role of epithelial-mesenchymal transition in cancer pathology. Pathology 39: 305-318, 2007.

25. Thiery JP, Acloque H, Huang RY and Nieto MA: Epithelialmesenchymal transitions in development and disease. Cell 139: 871-890, 2009. 